\title{
Integrating Site Conditions in a Web-based Intelligent Cost Estimator (WICE) System for Highway Construction Projects
}

\author{
Wen-der Yu, Gang-wei Fan, and Shao-Shung Lo
}

\begin{abstract}
This paper presents an integrated Web-based Cost Estimator (iWICE) system. The iWICE incorporates the influence of site conditions into a previous developed Web-based Intelligent Cost Estimator (WICE) system for highway construction projects. A VaFALCON neuro fuzzy system is employed to construct the site condition knowledge-based system that provide modifications for work unit costs from WICE. Moreover, VaFALCON allows user to input partial attribute information as complete attribute values are not available at the moment of estimation. The proposed iWICE system equips original WICE system with a more practical cost estimation system and provides more accurate estimates.
\end{abstract}

Index Terms-conceptual cost estimating, neuro-fuzzy system, site conditions, highway.

\section{INTRODUCTION}

$\mathrm{C}$ ONSTRUCTION cost estimation is critical not only for preparing the bids but also for selection of construction methods during project planning phase [1]. Previous effort of the research team has developed a Web-based Intelligent Cost Estimator (WICE) system for project participants to obtain project cost in a real-time basis [2]. The WICE system estimates construction cost based on the standard design drawings and specifications published by the Public Construction Commission of Taiwan Government. The site conditions of a specific construction project are found to have significant influence on construction costs. This research is a further step of WICE, which incorporates site-conditions of a specific project into the cost estimation process of a contractor. Thirty historical highway projects performed by the same general contractor are collected. Significant attributes that affect construction costs of a highway project are identified to

This work was financially supported by the National Science Council of Taiwan Government under Grant NSC 93-2622-E-216-013-CC3. Sincere appreciations are given from the authors to the sponsor

W. D. Yu, Associate Professor, Institute of Construction Management, Chung Hua University. Hsinhu, 30012, Taiwan (phone:+886-3-5186748; fax: +886-3-5370517; ( e-mail: wenderyu@ chu.edu.tw).

G. W. Fan, Master Student, Institute of Construction Management, Chung Hua University. Hsinhu, 30012, Taiwan (e-mail: me080kimo@yahoo.com.tw).

S. S. Lo, Ph.D. Student, Department of Technology Management, Chung Hua University. Hsinhu, 30012, Taiwan (e-mail: Kareh075@ms17.hinet.net). reflect the site conditions. A neuro-fuzzy system, VaFALCON, is employed to mine the estimation knowledge existing in the cost estimation data of the 44 historical projects. Eighty-one fuzzy IF-THEN rules are extracted to describe the estimation knowledge. The proposed VaFALCON provides not only explicit knowledge representation (fuzzy IF-THEN rules) but also a very desirable capability to handle incomplete information that usually exists in the historical data.

The rest of this paper is organized in the following manner: In the second section, previous literature on conceptual cost estimation are reviewed; the previously developed Web-based Intelligent Cost Estimator (WICE) system is revisited in the third section to provide a basis of this research; in the fourth section, previous effort on web agent (WA) for WICE system is described; in the fifth section, site conditions are incorporated into WICE to construct a more practical cost estimation system, where the most influential site attributes that influence highway construction costs are identified by interviews with the experienced construction managers/cost estimators, the historical project information along with the abovementioned parameters are collected, and data mining is performed using VaFALCON method. Real world highway construction projects are selected to test the proposed integrated system. Finally, conclusions are drawn and future works are recommended.

\section{LiterAtURE REVIEW ON CONCEPTUAL COST ESTIMATION}

There have been many conceptual methods proposed by previous researchers [1]-[13]. Many researchers have shown promising results in application of various AI techniques for construction conceptual costs estimation including applications of expert systems (ESs) [7], case-based reasoning (CBR) [8][9], artificial neural networks (ANNs) [10][11], and neuro fuzzy systems (NFSs) [12]. Others conducted researches on simulation and statistics regression approaches for cost estimation [5][9][13]. Most of the existing conceptual cost estimation methods were basically experience based, i.e., based on the domain experts' experience or knowledge.

Wilmot and Cheng [14] concluded three past methods for forecasting highway construction cost: (1) unit rates — such as dollars per mile; (2) extrapolation of past trend-collapsing 
various individual item costs to a single overall expression of construction costs for forecasting overall project cost; (3) parametric function models_-establishing functions to model the relationship between the construction cost and the influential factors. They proposed a "cost index method" for forecasting of highway construction costs. Wang and Mei [15] also developed construction cost indices for construction cost estimation of public works in Taiwan. Wilmot and Cheng pointed out that the cost index approaches for construction cost estimation fell short in incorporating all influential factors and producing project specific estimates. This is due to the unavailability of required information and the overall aggregation of cost indices.

$\mathrm{Yu}$ et al. [1] proposed a Web-based Intelligent Cost Estimator (WICE) system that integrated a Principal Items Ratio Estimating Method (PIREM) [3] with ANFIS neuro-fuzzy system [16] and internet information technology to form a real-time and globally accessible conceptual cost estimation system. Even though WICE was proved to be very useful for construction cost estimation in planning and design phase, it requires the user to input influential attribute values that are usually based on experienced engineer's judgment. Yu and Fu [17] developed a Web Agent (WA) to incorporate the experienced engineer's knowledge on attribute value judgments into WICE system. WA provides a facility for a user to assess attribute values of WICE.

\section{WEB-BASED INTELLIGENT COST ESTIMATOR (WICE)}

The WICE system was developed to meet the requirement of real-time cost estimation, under the collaboration efforts of Chung Hua University and the industrial partner, CHINA ENGINEERING CONSULTANTS, INC. (CECI), Taipei, Taiwan. The WICE system was constructed on three piers: (1) PIREM conceptual cost estimation model-a cost estimation method that separates unit price and quantity from the associated cost item, so that the most prevailing price information from market place can be reflected in the estimation; (2) ANFIS-a neuro-fuzzy system that provides the intelligence of WICE to learn work quantity knowledge of a cost item from its historical data; (3) the internet information technology-equips WICE with global accessibility and real-time responding functions.

\section{A. PIREM}

In WICE a Principal Items Ratio Cost Estimation Method (PIREM) [3] was adopted to take the "unit price" and "activity quantity" apart in an activity cost item. Previous knowledge acquired from historical data is used to estimate the quantities of an activity. On the other hand, the most updated unit price information is surveyed in the marketplace to reflect the prevailing unit prices of material, labor, and equipment. In order to simplify and reduce the estimation effort, only the most important cost items are taking into account for cost estimation. Thus, the Pareto Optimum Criterion (or named "80/20 Principle”) is adopted. Simply stated, the Pareto
Optimum Criterion suggests that $80 \%$ of the overall project cost is determined by $20 \%$ of the cost items. Therefore, instead of estimating the quantities of all cost items, only the top $20 \%$ most important cost items' quantities are estimated and their related unit prices are inquired. The selected top 20\% cost items is called "principal items $(P I)$ ". The summation of quantities and unit prices of the principal cost items constitutes the cost of principal items (PIC). The ratio of PIC over the overall cost is defined as the principal item ratio (PIR or $p$ ). The value of $p$ can be calculated by the following equation.

$$
p^{s}=\frac{\sum_{j=1}^{l} U P_{j}^{s} \cdot Q_{j}^{s}}{\sum_{i=1}^{n} U P_{i}^{s} \cdot Q_{i}^{s}}=\frac{P I C^{s}}{O C^{s}}
$$

In Equation (1), the numerator is the summation of the costs of all principal items, while the denominator is the summation of the costs of all cost items in a project. The super script $s$ upon all parameters stands for the $s^{\text {th }}$ historical example. $U P_{i}$ means unit price and $Q_{i}$ means quantity of the $i^{\text {th }}$ cost item, and so forth. $O C$ stands for overall cost. The ratio $p$ obtained in Equation (1) is call "principal item ratio (PIR)" as defined above.

It is found by analyzing PIR's obtained from historical cost estimation data that the PIR of a specific type of construction project usually keeps constant with very small variation. Therefore, given the PIC, the $O C$ of the new project can be recovered by the following equation.

$O C^{r}=\frac{P I C^{r}}{\bar{p}}$,

In Equation (2), $O C^{r}$ is the estimate of overall cost for the new $\left(r^{\text {th }}\right)$ project. $\bar{p}$ is the average PIR calculated from previous projects. $P I C^{r}$ is the cost of principal items of the new $\left(r^{\text {th }}\right)$ project

In Eq. (1) and (2), the current unit prices (UP's) for the new project should be inquired from the marketplace at the moment of estimation. While, the quantity, $Q$, and average $P I R, \bar{p}$, have to be determined by parametric estimating methods based on the pre-determined mathematic relationships. This relationship should be established according to the knowledge acquired from historical data.

\section{B. ANFIS}

In order to establish the relationship between influential attributes and activity quantities, a neuro-fuzzy data mining technique named ANFIS [16] was adopted to mine estimation knowledge from historical data. The architecture of ANFIS network consists of 6 layers: (1) Layer 0-input layer; (2) Layer 1-membership functions; (3) Layer 2-t-norm operation; (4) Layer 3-normalization of inference output; (5) Layer 4 Sugeno FIS (fuzzy inference system) operation; and (6) 
Layer 5-aggregation operation for output. By learning algorithms, ANFIS can be automatically constructed to provide same functions of a fuzzy inference system (FIS).

\section{Internet Implementation}

The Matlab ${ }^{\mathrm{TM}} 6.3$ with Web Server and Fuzzy Logic Toolboxes was adopted for WICE implementation. The framework and data flows of WICE are depicted in Fig. 1, where the Client (User) A submits parameter inputs (including influential attributes and unit prices) and job request to WICE by filling out the Parameter Input Sheet. The WICE then transmits the input data to Matlab ${ }^{\mathrm{TM}}$ main system via the interface and web-server programs. The Matlab ${ }^{\mathrm{TM}}$ main system consults related Matlab ${ }^{\mathrm{TM}}$ programs and performs the required calculations (including ANFIS network mapping), and then the results (including estimation results and relevant graphics) are generated. The numeric data (resulted estimates) and graphics are handled separately hereafter. The numeric data are transmitted backward via web-server and interface programs to the respond form of the Client. In the meanwhile, generated graphics (if applicable) are loaded on the respond form. Thus the request of Client $\mathrm{A}$ is completely served. The interface of WICE system is shown in Fig. 2and Fig. 3.

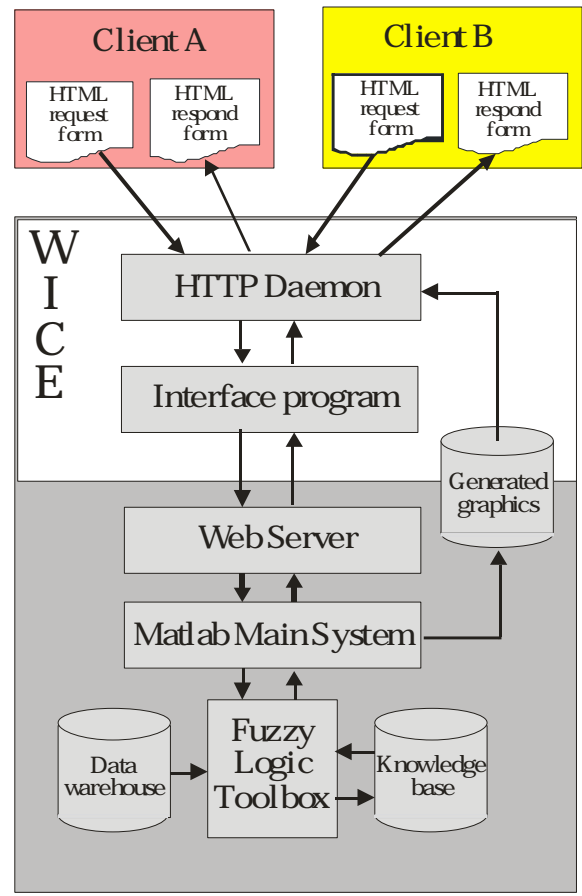

Fig. 1. Internet data flows of WICE [2].

There are eight modules in the WICE system for eight different types of work: (1) Reinforced concrete bridges; (2) Drainage works; (3) Earth moving; (4) Dike construction; (5) Retaining walls; (6) Slope protection works; (7) Pavement works; and (8) Building construction. Totally, 21 sub-modules were developed in WICE.

\section{Web Agent}

A Web Agent (WA) for highway construction cost estimation based on the WICE system was proposed by $\mathrm{Yu}$ and $\mathrm{Fu}$ [17]. The WA works like an experienced cost estimation consultant for the user. In developing WA, the cost estimation process for highway construction projects using WICE system was studied by interviews with domain experts of CECI. The process consists of three major steps: (1) collecting basic information regarding to the influential attributes based on the SPEC's and drawings; (2) estimating the values of influential attributes based on the judgments of domain experts or applying expert rules; (3) entering the values of influential attributes into WICE system and presenting the estimation results.

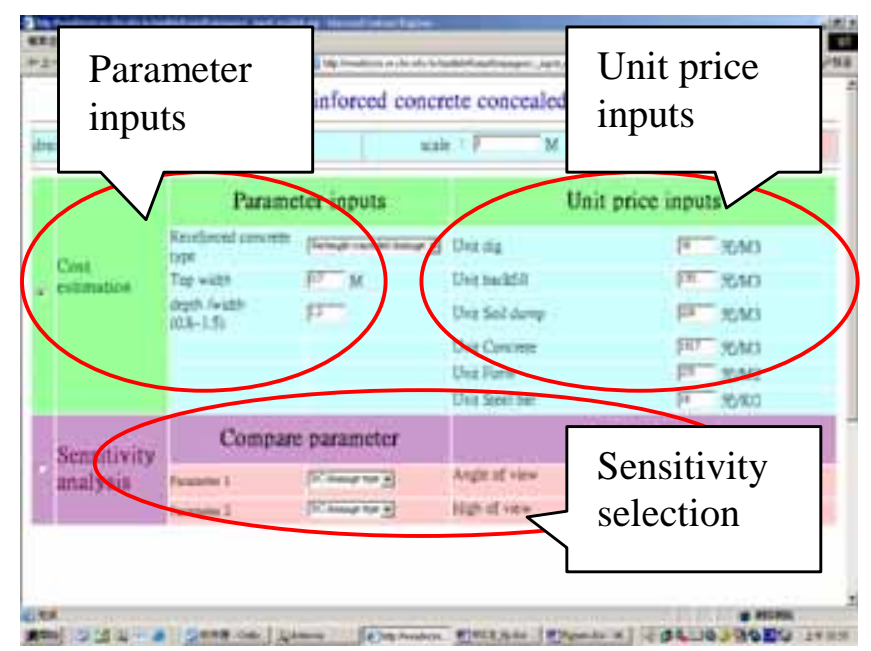

Fig. 2. Parameter Input Sheet of WICE [2].

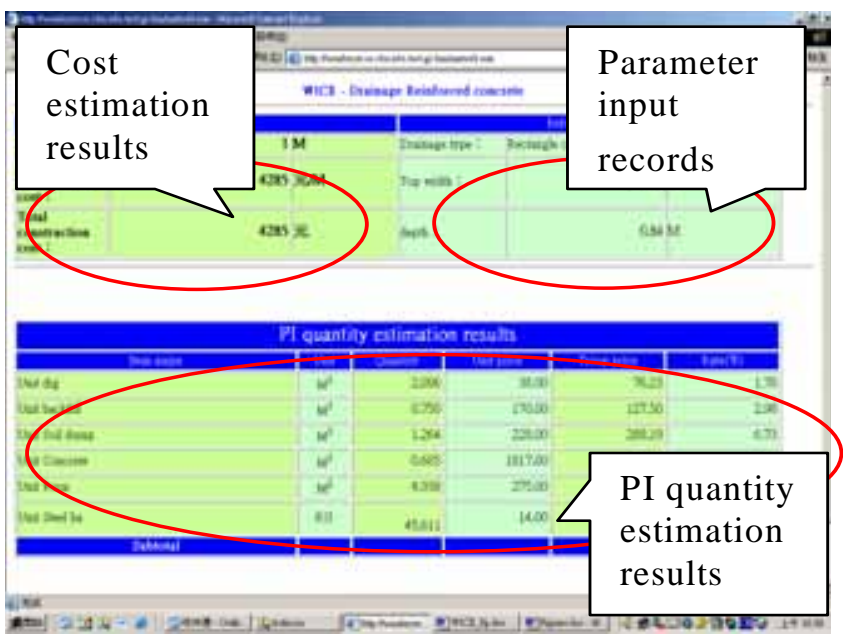

Fig. 3. Estimation Report Sheet of WICE [2].

The "intelligence" of the WA is the underlying knowledge stored in the knowledge base. In the WA, the knowledge of the domain experts is stored in terms of IF-THEN rules. In this research, totally 65 IF-THEN rues were generated through interviews with experiences estimators. These rules are stored in the form of decision tree. 
Framework of the proposed WAt system is shown in Fig. 4. The usage process of the proposed system consists of 6 steps: (1) assessment of attributes - by the process shown in Figure 3; (2) collaboration with various agents in WICE - to generate estimation results for each activities; (3) presentation of the estimation results to user; (4) evaluation of the estimation results by domain expert-if the results are approved, the estimation results are accepted and stored in the historical database as a new data, otherwise are expelled; (5) training of knowledge base - after a period of time, the knowledge base is updated by system training; (6) update of knowledge base of each agent.

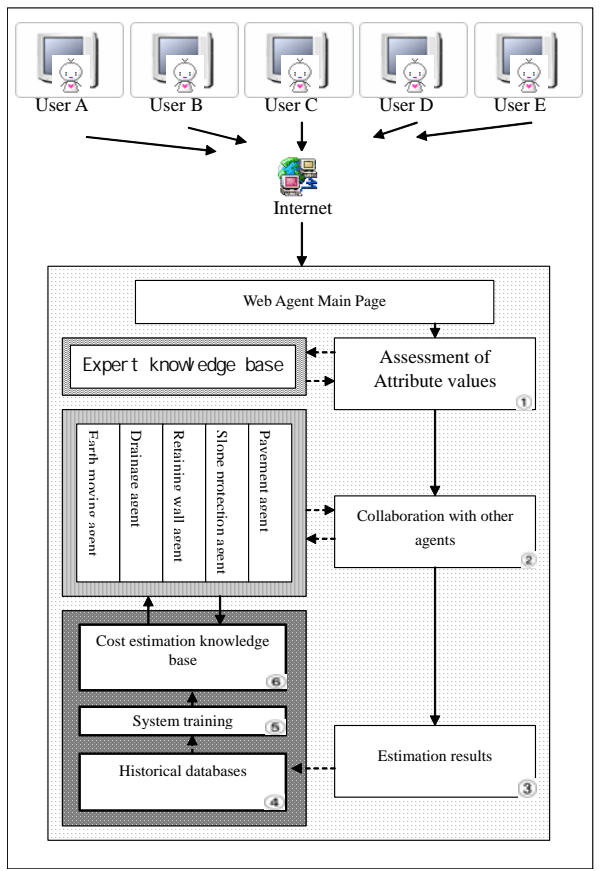

Fig. 4. System framework of WA [17].

\section{INCORPORATION OF SITE CONDITIONS}

\section{A. Identification of Influential Site Attributes}

The most significant site conditions that affect unit cost of highway construction work items were surveyed by interviews with the experienced managers/engineers of the industrial partner. For a highway construction project, the top four most significant attributes identified by research team are: (1) Traffic flow-the traffic flows affecting (crossing or parallel to the construction lanes) the construction sites; (2) Density of neighborhood - the crowdedness of the neighboring building; (3) Earth balancing - the extra effort required for earthmoving; (4). Method complexity-the complexity of the specific construction method required for the site. The list and fuzzy evaluation of the attributes is shown in Table I.
TABLE I

FuZZy EVALUATION OF KEy Site ATtRIBUTES

\begin{tabular}{ll}
\hline \hline \multicolumn{1}{c}{ Site attribute } & \multicolumn{1}{c}{ Fuzzy evaluation } \\
\hline Traffic flow (T) & HIGH, MEDIUM, LOW \\
Density of neighborhood (D) & HIGH, MEDIUM, LOW \\
Earth balancing (E) & HIGH, MEDIUM, LOW \\
Method complexity(M) & HIGH, MEDIUM, LOW \\
\hline
\end{tabular}

\section{B. Data Preparation}

A local general contractor who specialized in highway construction in the past 20 years was selected as the industrial partner for this project. Forty-four historical highway construction projects were provided by the industrial partner, including repaving and expanding of existing roads, construction of new roads, retaining walls, slope construction, and others.

According to the classification of WICE, six WICE modules are related: (1) Earth-moving module (EM); (2) Drainage module (DM); (3) Pavement module (PM); (4) Slope module; (5) Retaining-wall module (PM); and (6) Bridge module (BM). Not all of the above five modules are related to every historical project. Conceptual cost estimation was performed for the 44 historical projects by WICE. Historical unit cost of each work type and that estimated by WICE was recorded for every project. At the same time, the values of four key attribute describing the site conditions of the project were assessed and recorded. The result is a database recording the key attribute values and the associated ratios of historical unit cost over the estimated unit cost. The resulted database is shown in Table A.1 in the Appendix session.

\section{Data Mining}

A neuro fuzzy system named Variable-attribute Fuzzy Adaptive Logic Control Network (VaFALCON) [18] is adopted for mining of cost estimation knowledge of site condition effect. VaFALCON was evolved from the Fuzzy Adaptive Logic Control Network (FALCON) originally developed by Lin and Lee [19]. A standard FALCON consists of five layers. Each layer consists of nodes with proper numbers of fan-in and fan-out connections represented by weights assigned to the nodes. The fan-in connections connect the nodes of the previous layer with the nodes of the current layer. The fan-out connections connect nodes of the current layer with nodes of the subsequent layer. An integration function is associated with the fan-in connections of a node. The integration function can be a summation, activation, or fuzzy operation.

There are five layers in a FALCON: (1) Layer 1-input layer, which takes input attribute values from outside world; (2) Layer 2-input term nodes, which fuzzify the input attribute values into fuzzy variables; (3) Layer 3-rule nodes, which perform fuzzy AND operations; (4) Layer 4-output term nodes, which defuzzify the fuzzy functions concluded by the rule nodes; and Layer 5-output layer, which presents the system output to outside world.

Unlike original FALCON, VaFLACON adopts a Variable 
Attribute Network Structure (VANS) so that it is able to handle missing attribute values. This is very useful when some of the site condition information is not available at the moment of cost estimation. The system training and testing results are shown in Table II. In Table II, the five work type data were mined separately. As each historical highway construction project was related to one to five work types, the numbers of training and testing datasets for every work type were different. It is noted that the training accuracy ranges from $83.18 \%$ to $91.25 \%$, the testing accuracy ranges from $82.07 \%$ to $90.18 \%$. Since the fuzzy partition for the key site attributes is [ [ $\left.\begin{array}{llll}3 & 3 & 3 & 3\end{array}\right]$ as shown in Table I, the resulted VaFALCON is equivalent to a fuzzy inference system (FIS) with $3 \times 3 \times 3 \times 3=81$ fuzzy IF-THEN rules for each work type.

TABLE II

TRAINING AND TESTING RESUlTS OF DATA MINING

\begin{tabular}{ccccc}
\hline \hline Work & \multicolumn{2}{c}{ Training Set } & \multicolumn{2}{c}{ Testing Set } \\
Type & No. of Data & Accuracy & No. of Data & Accuracy \\
\hline EW & 31 & $91.11 \%$ & 6 & $90.18 \%$ \\
DW & 29 & $83.18 \%$ & 5 & $83.96 \%$ \\
PW & 33 & $87.97 \%$ & 6 & $90.09 \%$ \\
BW & 11 & $87.74 \%$ & 2 & $89.38 \%$ \\
RW & 30 & $88.13 \%$ & 6 & $82.07 \%$ \\
SW & 26 & $91.25 \%$ & 6 & $85.54 \%$ \\
\hline \hline
\end{tabular}

Work Type: EW=earthmoving work, DW=Drainage work, PW=Pavement work, BW=Bridge work, RW=Retaining-wall work, SW=Slope work.

\section{Integrated System Framework}

The proposed integrated cost estimation system that incorporates site condition effect and web agent for input attributes into WICE system is called Integrated Web-based Cost Estimator (iWICE). The framework of iWICE is depicted in Fig. 5.

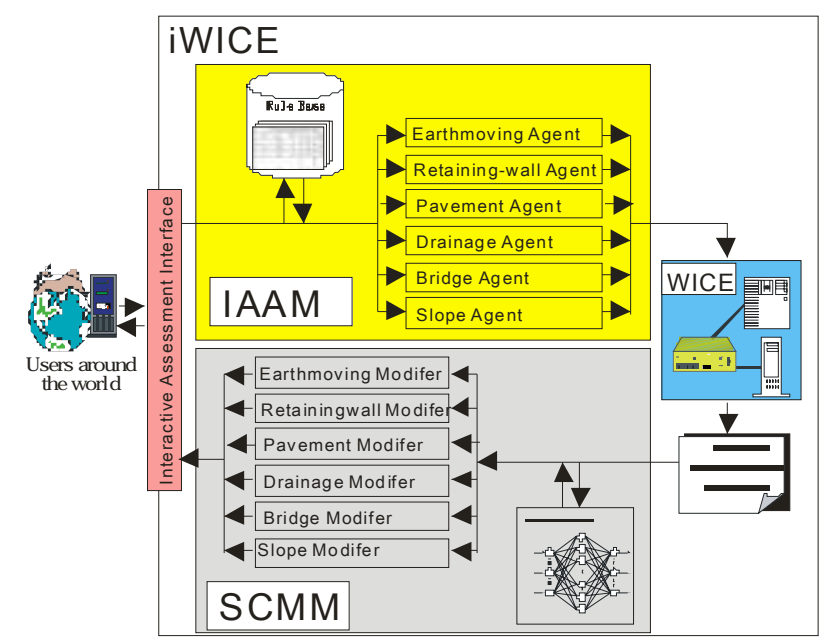

Fig. 5. Framework of iWICE

The proposed iWICE system consists of four main modules: (1) Interactive Assessment Interface-communicating with the user via interactive web interface; (2) Input Attribute Assessment Module (IAAM)—assessing input attribute values by consulting the expert rule base; (3) Web-based Intelligent Cost Estimator (WICE)-performing conceptual cost estimation; and (4) Site Condition Modification Module (SCMM)-modifying the estimates from WICE by consulting VaFALCON neuro fuzzy system.

\section{DEMONSTRATION}

In this section, a real world highway construction project is selected to demonstrate the application of the iWICE system.

\section{A. Case Description}

The selected highway project is the road improvement project connecting Shiang-Shan Interchange of the Second Freeway of Taiwan to Provincial Highway No. 13, from $4 \mathrm{k}+580$ to $5 \mathrm{k}+618$. Some project information is as follows:

- Construction cost: TWD 18,000,000 (USD 573,248);

- Project duration: 180 calendar days;

- Length of road: $1.038 \mathrm{~km}$;

- Width of road: $20 \mathrm{~m}$;

- Site classification: $80 \%$ plane; $20 \%$ hill;

- $\quad$ Distance to earth dump area: $5 \mathrm{~km}$;

- $\quad$ Speed for traveling to dump: $40 \mathrm{~km} / \mathrm{hr}$.;

- Thickness of asphalt layer: $15 \mathrm{~cm}$;

- Thickness of gravel layer: $35 \mathrm{~cm}$.

\section{B. Input Attribute Assessment}

There are three work types related to this project: (1) Earth moving work; (2) Drainage work; and (3) Pavement work. In order to assess the required attribute values, extra information is requested by IAAM of iWICE:

- Earth moving work-“"“Lane width” = $20 \mathrm{~m}$; "Site classification" $=80 \%$ plane $+20 \%$ hill; "Length” = $1.038 \mathrm{~km}$.

- Drainage work—“Dump distance" = $5 \mathrm{~km}$; "Influx area" = 20 hectare;

- Pavement work—Road class" = provincial highway; "Lane width" $=20 \mathrm{~m}$.

After consulting the rule base of IAAM, the input attribute values for each work type are: (1) Earth moving work-“Dump distance'= $5 \mathrm{~km}$, "Speed" = $40 \mathrm{~km} / \mathrm{hr}$.; (2) Drainage work - "Drainage type" = open channel with width $=30 \mathrm{~cm}$ and depth/width = 1.3; and (3) Pavement work- "Asphalt thickness"= $15 \mathrm{~cm}$, "Gravel thickness" = 35 cm, "Height" = $3 \mathrm{~m}$, "Width" = $20 \mathrm{~m}$.

\section{Unit Prices of Principal Items}

The prevailing unit prices of principal items for each work type are surveyed from the marketplace: (1) Earth moving work-cleaning=TWD $4 / \mathrm{m}^{2}$, embankment= TWD $24 / \mathrm{m}^{3}$, borrowing $(10 \mathrm{~km})=180 / \mathrm{m}^{3}$; (2) Drainage work-excavation work=TWD $30 / \mathrm{m}^{3}$, refilling $=$ TWD $8 / \mathrm{m}^{3}$, ready-mixed concrete=TWD $1300 / \mathrm{m}^{3}$, rebar=TWD $2.5 / \mathrm{kg}$; and (3) Pavement work-aggregate material=TWD $320 / \mathrm{m}^{3}$, ready mixed asphalt concrete $=$ TWD 845/ton, asphalt $($ AC-20 $)=$ TWD 4/kg. 


\section{Key Site condition Attribute Assessment}

The four key site condition attributes are assessed by SCMM. The assessment results are shown in Table III, where the method complexity is "MEDIUM" since two drainage types were used in this project within $1 \mathrm{~km}$. The earth is well balanced. No extra earthmoving work is required. The construction site is located at a traffic busy area, where surrounding buildings are crowded. The modifiers estimated by input the key site condition attribute values to VaFALCON are shown in Table IV. The adjusted total construction cost for this project is TWD 17,983,667 (USD 572,728). The actual cost is TWD 18,000,000 (USD 573,248). The estimation error before modification is TWD 16,333 (0.09\%). After adjustment with modifiers, the estimation error before modification is TWD 348,320 (1.94\%). The estimation accuracy is significantly improved.

TABLE III

AsSESSMENT RESUlts OF Key Site ATtRIBUTES

\begin{tabular}{lc}
\hline \hline \multicolumn{1}{c}{ Site attribute } & Fuzzy evaluation \\
\hline Traffic flow (T) & $H I G H$ \\
Density of neighborhood (D) & $H I G H$ \\
Earth balancing (E) & LOW \\
Method complexity(M) & MEDIUM \\
\hline
\end{tabular}

TABLE IV

VALUES OF MODIFIERS

\begin{tabular}{crrr}
\hline \hline Work Type & $\begin{array}{c}\text { Estimated cost } \\
\text { by WICE }\end{array}$ & $\begin{array}{l}\text { Modifier } \\
\text { values }\end{array}$ & Adjusted Cost \\
\hline Earth moving work & $1,097,760$ & 1.1004 & $1,207,975$ \\
Drainage work & 983,920 & 1.1890 & $1,169,881$ \\
Pavement work & $15,570,000$ & 1.0023 & $15,605,811$ \\
Sum & & & $17,983,667$ \\
\hline \hline
\end{tabular}

\section{CONCLUSION AND RECOMMENDATIN}

This paper presents a web-based integrated highway construction cost estimation system named Integrated Web-based Cost Estimator (iWICE) system. The iWICE is based on a previously developed web-based commercial cost estimation system, WICE, which provides globally accessible and 24-hour real-time cost estimating functions. The proposed iWICE system improves the original WICE in two ways: (1) facilitating the user in assessing the input attribute values that usually base on the engineering judgments of experienced managers/engineers; (2) modifying the estimates of WICE by considering site condition effects. In addition to the above functions, iWICE system retains the merits of the original WICE including lowest maintenance costs, global and all-time accessibility, and real-time response.

Future directions after this research may include (1) automated learning process of expert knowledge base for acquiring information provided by the users in IAAM; (2) incorporating more key site condition attributes in SCMM to improve estimation accuracy; (3) extending iWICE to mobile business.

\section{APPENDIX}

TABLE A.I

UNIT COST RATIOS OF HISTORICAL/ESTIMATED DATA

\begin{tabular}{|c|c|c|c|c|c|c|c|c|c|c|}
\hline \multirow[t]{2}{*}{ ID } & \multicolumn{4}{|c|}{$\begin{array}{c}\text { Fuzzy Evaluation of } \\
\text { Key Site Attribute }\end{array}$} & \multicolumn{6}{|c|}{$\begin{array}{l}\text { Historical/Estimated Unit Cost Ratio } \\
\text { for Work Type }\end{array}$} \\
\hline & $\mathrm{T}$ & $\mathrm{D}$ & $\mathrm{E}$ & $\mathrm{M}$ & EW & DW & PW & BW & RW & SW \\
\hline 1 & $\mathrm{M}$ & $\mathrm{H}$ & $\mathrm{L}$ & $\mathrm{H}$ & 0 & 0 & 1.0 & 0 & 0 & 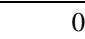 \\
\hline 2 & $\mathrm{H}$ & $\mathrm{H}$ & $\mathrm{L}$ & $\mathrm{H}$ & 0 & 0 & & 0 & 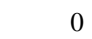 & O \\
\hline 3 & M & M & $\mathrm{L}$ & M & 0.8033 & 1.1213 & 1.0536 & 0 & 133 & 14 \\
\hline 4 & M & $\mathrm{H}$ & M & M & 1.1030 & 1.2177 & 0.9366 & 0 & 0 & \\
\hline 5 & $\mathrm{~L}$ & $\mathrm{~L}$ & $\mathrm{~L}$ & M & 0.5648 & 0 & 0 & 0 & 1.1311 & 0 \\
\hline 6 & $\mathrm{~L}$ & M & $\mathrm{L}$ & M & 1.2353 & 1.3066 & 0.9636 & 0 & 1.1301 & \\
\hline 7 & $\mathrm{~L}$ & M & $\mathrm{L}$ & M & 1.0230 & 0.9110 & 1.0121 & 0 & 1.2488 & 1.1824 \\
\hline 8 & M & M & M & M & & & & 0 & & \\
\hline 9 & $\mathrm{~L}$ & $\mathrm{~L}$ & $\mathrm{~L}$ & M & 0.0000 & 0 & 1.0 & 0 & 0 & 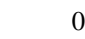 \\
\hline 10 & $\mathrm{~L}$ & M & M & M & 0.8327 & 0 & & 0 & 1.0001 & م \\
\hline 11 & $\mathrm{~L}$ & $\mathrm{~L}$ & M & $\mathrm{L}$ & 1.1833 & 1.5232 & 1.1877 & 0 & 1.2633 & \\
\hline 12 & M & M & $\mathrm{L}$ & M & 0.0000 & 0 & 0.9 & 0 & 0 & 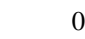 \\
\hline 13 & M & $\mathrm{L}$ & $\mathrm{L}$ & M & 0.0000 & 0 & & 0 & 0 & 0 \\
\hline 14 & $\mathbf{M}$ & M & $\mathrm{L}$ & M & & 1.2 & 0 & & 0.9678 & 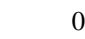 \\
\hline 15 & $\mathrm{~L}$ & $\mathrm{~L}$ & $\mathrm{H}$ & $\mathrm{L}$ & & & 0 & 0 & & 360 \\
\hline 16 & M & M & $\mathrm{L}$ & M & & & & 0 & & \\
\hline 17 & M & M & $\mathrm{L}$ & M & 1.1623 & 1.0383 & 0.8 & 0 & 1.1 & 1.0080 \\
\hline 18 & M & M & M & M & & 1.0 & & 0 & & 1.1911 \\
\hline 19 & $\mathrm{H}$ & $\mathrm{H}$ & M & M & & 1.0766 & 1.0 & 0 & 1. & 1.0635 \\
\hline 20 & M & $\mathrm{H}$ & $\mathrm{L}$ & M & & 1.2 & 1.1 & 0 & & \\
\hline 21 & M & $\mathrm{H}$ & $\mathrm{L}$ & M & 1.4 & 1.1 & 1.0 & 0 & 76 & \\
\hline 22 & 10 & M & 11 & M & & & & 0 & & 1.0376 \\
\hline 23 & M & M & M & M & $1 .($ & 0.9 & & 0 & 17 & 1.2497 \\
\hline 24 & $\mathrm{~L}$ & $\mathrm{~L}$ & M & M & & 1.2 & & 0 & & 1.0102 \\
\hline 25 & $\mathrm{~L}$ & M & M & M & 1.2 & 0.9 & 1.0 & 63 & 1. & 1.2956 \\
\hline 26 & $\mathrm{~L}$ & $\mathrm{~L}$ & M & M & 1.4 & 1.0989 & 1.1 & 0.9638 & 1.2923 & 1.3015 \\
\hline 27 & $\mathrm{~L}$ & M & M & $\mathrm{L}$ & & & & & & \\
\hline 28 & $\mathrm{~L}$ & $\mathrm{~L}$ & $\mathrm{~L}$ & M & & 0.9 & 1.1 & 1.3696 & & 1.2007 \\
\hline 29 & $\mathrm{~N}$ & M & M & M & & & & & & \\
\hline 30 & $\mathrm{~L}$ & M & M & $\mathrm{L}$ & & 1.0 & 0.9 & 1.2997 & 1. & 1.1020 \\
\hline 31 & M & $\mathrm{H}$ & M & M & $1 .($ & 1. & 0.5 & 0 & 65 & 0.9345 \\
\hline 32 & M & M & $\mathrm{H}$ & M & & & & 0 & & 1.0899 \\
\hline 33 & $\mathrm{~L}$ & $\mathrm{H}$ & H & M & & 1.2101 & 0.9 & 0 & & 1.1007 \\
\hline 34 & $\mathrm{H}$ & $\mathrm{H}$ & M & M & 1.2983 & 1.0998 & 1.0876 & 1.3003 & 1.1010 & 1.0997 \\
\hline 35 & $\mathrm{~L}$ & M & M & $\mathrm{L}$ & 1.4067 & & 1.0947 & 0 & 1.2988 & 1.4031 \\
\hline 36 & M & M & M & $\mathrm{L}$ & 1.1007 & 0.9927 & 1.0 & 1.3011 & 1.1890 & 1.0056 \\
\hline 37 & $\mathrm{~L}$ & M & M & M & 0.8003 & 0.9968 & 0.9972 & 0 & 1.0002 & 0.5023 \\
\hline 38 & $\mathrm{~L}$ & $\mathrm{~L}$ & $\mathrm{H}$ & $\mathrm{L}$ & & 0 & 1.0231 & 0 & 0 & 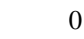 \\
\hline 39 & M & M & $\mathrm{L}$ & M & 1.2943 & 1.2986 & 0.9728 & 1.1979 & 1.2050 & 1.3003 \\
\hline 40 & $\mathrm{~L}$ & $\mathrm{H}$ & M & M & 0.0000 & 0 & 0.9937 & 0 & 0 & 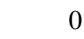 \\
\hline 41 & M & $\mathrm{L}$ & $\mathrm{L}$ & M & 1.1866 & 1.1986 & 0.9578 & 1.1034 & 1.1968 & 0.9937 \\
\hline 42 & M & M & M & M & 1.0873 & 0 & 0 & 0 & 1.3015 & 1.2080 \\
\hline 43 & M & $\mathrm{H}$ & $\mathrm{L}$ & M & 0.9863 & 1.0370 & 0 & 0 & 1.0010 & 1.2897 \\
\hline 44 & $\mathrm{~L}$ & $\mathrm{~L}$ & $\mathrm{H}$ & $\mathrm{L}$ & 1.1963 & 1.0323 & 1.0093 & 0 & 1.1210 & 1.0078 \\
\hline
\end{tabular}

Key Site Attribute: T=Traffic flow, D=Density of neighborhood, E=Earth balancing, $\mathrm{M}=$ Complexity of method.

Fuzzy Evaluation: L=Low, M=Medium, H=High.

Work Type: EW=earthmoving work, DW=Drainage work, PW=Pavement work. BW=Bridge work. RW=Retaining-wall work. SW=Slope work.

\section{ACKNOWLEDGMENT}

The authors sincerely appreciate the sponsor, National Science Council of Taiwan Government, for providing financial support for this research.

\section{REFERENCES}

[1] W. D. Yu and C. C. Lai, "WICE: A Web-based Intelligent Cost Estimator for Real-time Decision Support,” Proceedings of IEEE/WIC 
International Conference on Web Intelligence (WI'03), Oct. 13 16, Session 10B, October 13-16, 2003, Lord Nelson Hotel, Halifax, Canada, pp. 646 649, 2003.

[2] W. D. Yu, C. C. Lai, and W. L. Lee, "A WICE Approach to Real-time Construction Cost Estimation," Automation in Construction, 10 pp., 2005. (in press)

[3] W. D. Yu, "PIREM: a new model for conceptual cost estimation," Construction Management and Economics, 29 pp., 2005. (in press)

[4] A. Akintoye and Fitzgerald, E., "A survey of current cost estimating practices,” Construction Management and Economics, Vol. 18, No. 2, pp. 161-172, 2000.

[5] N. A. Koenigseker, "Parametric estimating of buildings," Cost Engineering, Vol. 24, No. 6, 1982, pp. 327-332.

[6] S. Karshenas, "Predesign cost estimating method for multistory building," Journal of Construction Engineering and Management, ASCE, Vol. 100, No. 1, pp. 79-86, 1984.

[7] F. Khosrowshahi and Kaka, A. P., "Estimation of project total cost and duration for housing projects in the UK," Building and Environment, Vol. 31, No. 4, pp. 373-383, 1996.

[8] J. S. Yu, "Developing building cost estimating system using case-based reasoning approach,” Master Thesis, Department of Civil Engineering, National Central University, Chungli, Taiwan, R.O.C., 2001.

[9] G. H. Kim, S. H. An, and K. I. Kang, "Comparison of construction cost estimating models based on regression analysis, neural networks, and case based reasoning," Building and Environment, Vol. 39, No. 10, pp. 1235-1242, 2004.

[10] G. H. Kim, Yoon, J. E., S. H. An, H. H. Cho, and K. I. Kang, "Neural network model incorporating a genetic algorithm in estimating construction costs,” Building and Environment, Vol. 39, No. 11, pp. 1333-1340, 2004.

[11] T. Hegazy and A. Ayed, "Neural network model for parametric cost estimation of highway projects," Journal of Construction Engineering and Management, ASCE, Vol. 124, No. 3, pp. 18-24, 1998.

[12] W. D. Yu and J. B. Yang, "Data Mining for the Cost Estimating of Highway Bridges Construction with a Neuro-Fuzzy System”, Proceedings of 2001 Ninth National Conference on Fuzzy Theory and Its Applications, Nov. 23 24, National Central University, Chung-li, Taiwan, pp. 437 442, 2001.

[13] A. J. Vergara and L. T. Boyer, "Probabilistic approach to estimating and cost control," Journal of Construction Division, ASCE, No. 100, pp. 543-552, 1974.

[14] C. G. Wilmot and G. Cheng "Estimating future highway construction costs,” Journal of Construction Engineering and Management., Vol. 129, No. 3, pp. 272-279, 2003.

[15] C. H. Wang and Y. H. Mei, "Model for forecasting construction cost indices in Taiwan," Construction Management and Economics, Vol. 16, No. 2, pp. 147-157, 1998.

[16] J. S. Jang, "ANFIS: Adaptive-network-based fuzzy inference system," IEEE Transactions on Systems, Man, and Cybernetics, Vol. 23, No. 3, 1993, pp. 665-685.

[17] W. D. Yu,and J. W. Fu, "A Web Agent for Conceptual Cost Estimation of Highway Construction Projects,” Proceedings of ISARC 2004, Session S19-05, Sep. 21 25, Jeju, Korea, pp. 658-663, 2004.

[18] W. D. Yu and H. W. Lin, "A VaFACON neuro fuzzy system for mining of incomplete construction databases," Automation in Construction, 2005, 13 pp. (in press)

[19] C. T. Lin and C. S. G. Lee, "Neural-network-based fuzzy logic control and decision system,” IEEE Transactions on Computers, Vol. 40, No. 12, pp. 1320-1336, 1991. 